\title{
Editorial
}

\section{Acta Neuropsychiatrica and Scandinavian College of Neuropsychopharmacology (SCNP)}

With this editorial, we are happy to announce that Acta Neuropsychiatrica becomes the official journal of the Scandinavian College of Neuropsychopharmacology $(S C N P)$, which is among the oldest neuropsychopharmacological associations in the world. SCNP was founded in 1959 on the initiatives of David H. Ingvar, Jørgen Ravn and Arvid Carlsson. The association had the first meeting in Copenhagen in 1960, and, with annual meetings, SCNP could in 2009 in Copenhagen celebrate its 50th annual meeting anniversary, which on that occasion were held as a joint meeting with the Canadian College of Neuropsychopharmacology.

In the past 50 years, $S C N P$ has fostered educational initiatives with impact not only in the Scandinavian countries but also worldwide. Several remarkable scientific observations have been carried out by the members of $S C N P$. It is not possible here to highlight all high-impact discoveries, but the work by the first SCNP President and Nobel Laureate Arvid Carlsson should be mentioned here. Arvid Carlsson applied with great success the results from basic research into the clinical field, most clearly illustrated by the understanding of the importance of dopamine in several psychiatric conditions, as well as his pioneer development of the second-generation antidepressants, the selective serotonin reuptake inhibitors (SSRIs). Also worth to mention is the work by Mogens Schou, SCNP board member and Lasker Awardee, who brought lithium prophylaxis from experimental therapy to routine treatment, thereby helping thousands of patients. For a short overview of some of the major achievements made by SCNP during the first 25 years, see the review by C. G. Gottfries (1). Also relevant to the neuropsychiatric research area is the work performed by a core committee within $S C N P$, which during several years worked on the characterisation of side effects following psychotropic medication.
The efforts of this work have resulted in a comprehensive rating scale tool, the UKU Side Effect Rating Scale (2), http://scnp.org, which now is widely used in both academia and industry. Finally, during the 25th annual meeting of SCNP held in Copenhagen in 1984, the first founding steps of formation of the European College of Neuropsychopharmacology were taken (3).

With this brief historical overview, we are enthusiastic about the future and progress of Acta Neuropsychiatrica. It is therefore a great privilege here to invite all members of SCNP to contribute and publish in Acta Neuropsychiatrica. Importantly, however, neuropsychiatry is a global field, and we emphasise that contributions from all over the world are welcome. The overall purpose of Acta Neuropsychiatrica remains to provide our readers with the latest perspectives from current and especially emerging areas, across all disciplines and fields relevant to neuropsychiatry.

This issue begins with a comprehensive and relevant review of obstructive sleep apnea and schizophrenia, where a very important role of the psychiatrist is pointed out (4). Thereafter follows a small review and case report on the treatment for psychosis during pregnancy (5). The remaining part of the issue focuses at depression and bipolar disorders from various perspectives. Commentaries on the published content are as usual welcome.

Gregers Wegener Editor-in-Chief, Centre for Psychiatric Research, Aarhus University, Aarhus, Denmark

Ole A. Andreassen President, SCNP Oslo University Hospital \& University of Oslo Norway 


\section{Editorial}

\section{References}

1. Gottfries CG. The Scandinavian Association of Psychopharmacology - 25 years. Nord J Psychiatry 1984;38: 29-32.

2. Linguaerde O, Ahlfors UG, Bech P, Dencker SJ, ELGEN K. The UKU side effect rating scale. A new comprehensive rating scale for psychotropic drugs and a cross-sectional study of side effects in neuroleptic-treated patients. Acta Psychiatr Scand Suppl 1987;334:1-100.

3. Bech P. Scandinavian Association of Psychopharmacology 1959-1984. Nord J Psychiatry 1984;38:3.

4. Alam A, Chengappa KNR. Obstructive sleep apnoea and schizophrenia: a primer for psychiatrists. Acta Neuropsychiatrica 2011;23:201-209.

5. NiELSEN R. Treatment of psychosis during pregnancy - a case report and a mini-review. Acta Neuropsychiatrica 2011;23:210-214. 